\title{
Transferts thermiques convectifs en mini-canal : confrontation calculs/mesures
}

\author{
Convective heat transfer in mini-channel: \\ comparison between measurements and computational results
}

\author{
Sylvain Reynaud', Jean-Pierre Franc, Thierry Maître \\ Laboratoire des Ecoulements Géophysiques el Industriels (Grenoble)
}

François Debray

Laboratoire des Champs Magnétiques Intenses (Grenoble)

We present a series of measurements of the friction factor and the forced convection heat transfer coefficient in 2D mini-channels of $1.12 \mathrm{~mm}$ to $300 \mu \mathrm{m}$ in thickness. The friction factor is estimated from the measurement of the pressure drop along the whole channel. The heat transfer coefficient is determined from a local and direct measurement of the temperature and the heat flux at the wall by means of a specific transducer. The experimental results do not lead to question the validity of the classical correlations obtained on ducts of larger size. The numerical simulations have shown that the deviations observed from the classical laws for both the friction factor and the Nusselt number are mainly due to macroscopic effects as entrance effects or the lack of uniformity of the surface heat flux.

\section{INTRODUCTION}

Le Lahoraloire des Ecoulements Géophysiques el Industriels mène. en collahoration avec le Laboratoire des Champs Magnétiques Intenses. une élude sur les transferts thermiques par convection forcée en mini-canaux. Elle a pour but l'optimisation thermo-hydraulique des aimants à champs intenses du LCMI. Du fail de la géométrie simple de la veine d'essai. cette étude présente un caractère fondamental qui élargit le champ d'investigation initial à la question de la validité des corrélations classiques établies généralement pour des conduiles de taille bien supérieure. Une première série de mesures [I] a mis en évidence des comportements du nombre de Nusselt el du coefficient de frottement globalement proches des lois classiques. comple tenu des incestitudes de mesure. Une partie de l'instrumentation el du protocole expérimental a été modifiée suivant les enseignements lirés de cette première série. Une étude numérique de la veine d'essai a également élé menée en parallèle.

Un grand nombre de travaux. menés au cours des vingl demières années. sur les écoulements et les transferts thermi-

1. LEGI-ENSHMG, Domaine Liniversilaire, BP 53. 38041 Grenoble cedex 9 - sylviıiл.reynaud@hmg.inpg.tr ques en micro et mini-čanaux ont déjì fait l'objet de publication. [ls ont mis en évidence des déviations importantes par rapport aux corrélations couramment utilisées el présentent des conclusions parfois contradicloires. Les principaux résultals de cerlaines éludes de transferts thermiques en minicanaux reclangulaires (épaisseur $\geq 100 \mu$ lul sont ici présentés. Rahman [2] a obtenu des valeurs de Nu supérieures à la théorie en régime laminaire el lurbulent ${ }^{2}$. Ses résultats ne meltent pas en évidence un nombre de Reynolds de transition différent de celui altendu ( $\approx 2360$ ) et les valeurs du coefficient de frottement $C_{f}$ pour le canal le plus mince $\left(D_{H} \approx 360 \mu \mathrm{m}\right)$ sont conformes aux corrélations vałables pour un écoulement laminaire non-élabli dans un cube. Les Iravaux de Adams e't al. [3] vom également en ce sens puisqu'ils obliennent des nombres de Nusselt supérieurs à ceux donnés par la corrélation de Gnielinski $i^{3}$ dans un canal cylindrique ${ }^{4}$.

En revanche Gao el ol. [4] dans un canal rectangulaire obliennent des valeurs de $N_{u}$ inférieures à celles prévues par la théorie pour $D_{H} \leq 0,77 \mathrm{~mm}$ en régime laminaire el turbu-

2. $3(x) \leq /\}_{N}(H n) \leq 5(x), R c \leq 3 \mathrm{COU}$. Iluide Étudié : calt.

$3.2300 \leq R_{t} \leq 10^{6}, 0,5 \leq P_{r} \leq 2000$.

4. $0.76 \leq D(\mathrm{~mm}) \leq 1,09.3200 \leq$ Re $\leq 23000$, fluide étudic : cau.

5. $2(X)$ u $5 D_{\| \prime} \leq 1.82 \mathrm{~mm} .200 \leq R_{e} \leq 8$ UUO. Iluide étudié : cau. 
lent. Le coefficient de frottement suit les lois classiques en régime laminaire comme en régime turbulent, quel que soit le diamè̀re du canal. La transition à la turbulence a lieu à des nombres de Reynolds conformes aux valeurs classiquement admises (2300-4000). On peut citer les travaux de Wang \& Peng [5] et Peng \& Peterson [6] sur des multicanaux parallèles de section rectangulaire ${ }^{6}$. En régime laminaire comme en régime turbulent, le nombre de Nusselt est inférieur aux corrélations classiques. De plus il croît avec le nombre de Reynolds en régime laminaire alors que la théorie pour les conduites classiques prévoit une valeur constante. Ils ont observé la transition à la turbulence à des nombres de Reynolds d'environ 700-\$000 et la turbulence développée vers I 500.

Les travaux de Acosta et al. [7] s'attachent plus spécifiquement au coefficient de frottement. Ils utilisent plusieurs canaux rectangulaires ${ }^{7}$ présentant des mgosités de type industriel différentes. Un canal supplémentaire est étudié. Ses parois ont été particulièrement soignées ${ }^{8}$ pour atteindre un état de surface dit «poli optique». En régime laminaire, l'ensemble des mesures est proche de la loi de HagenPoiseuille: $C_{f}=16 / R e$. En régime turbulent, bien que les écoulements pour l'ensemble des canaux correspondent théoriquement au régime lisse. les auteurs ont observé que seul le cas du «poli oplique » suivait la loi $C_{f}=0.079 . R e^{-0.25}$. Les mesures faites dans les canaux ayant des nıgosités plus élevées s'écartent sensiblement vers des valeurs supérieures à celles attendues. Par ailleurs, le changement de régime intervient à des nombres de Reynolds conventionnels $(-2500)$. Ce demier point est en contradiction avec les travaux de Zeighami es al. [8] qui ont observé la transition à la turbulence pour $1200 \leq \operatorname{Re} \leq 1600$ dans un canal de section rectangulaire $\left(D_{H}=120 \mu\right.$ !) $)$.

En résumé. les études mentionnées s’accordent sur le fait que le coefficient de frottement ne subit pas de déviation significative par rappont aux lois classiques pour les minicanaux de diamètre hydraulique supérieurs à $200 \mu \mathrm{m}$ du moment que l'on porte une attention particulière à la mgosité de paroi. En revanche les conclusions quuant au nombre de Nusselt sont contradictoires. Les études font état de valeurs supérieures ou inférieures aux corrélations classiques, en régime laminaire comme en régime turbulent. Il en est de méme pour le nombre de Reynolds de transition puisqu'il s'échelonne des grandeurs classiques à des valeurs bien plus faibles.

\section{D DISPOSITIF EXPÉRIMENTAL}

\section{Il.I Boucle et veine d'essais}

La boucle hydrodynamique est composée de deux cuves de 450 litres chacune contenant de l'eau de ville filtrée ${ }^{9}$ et mise en circulation par une pompe centrifuge. Les conduites situées entre les deux cuves ainsi que la veine d'essais sont isolées thesmiquement de l'extérieur par des panneaux de laine de roche de $10 \mathrm{~cm}$ d'épaisseur.

La cellule d'essais se présente sous la forme d'un support en bronze de section rectangulaire $\left(370 \times 150 \times 80 \mathrm{~mm}^{3}\right)$

6. $4.74 \geq D_{H}(\mathrm{~mm}) \geq 0,31$. Nuides ćludiés : cau. méthanol.

7. $D_{t}=960 \mathrm{~km}, \mathrm{l} 300 \leq$ Re $\leq 22000$, fluide étudie : solution aqueuse.

8. Polissige au moyen d'une poudre de diamant de $0.1 \mu \mathrm{m}$.

9. La dimensien caractéristique des mailles des tiltres est 5 jun. dans lequel un canal plan a été usiné $\left(60 \times 20 \mathrm{~mm}^{2}\right)$. Trois hublots interchangeables de forme convergente/divergente créent un mini-canal plan de $60 \mathrm{~mm}$ de largeur. $150 \mathrm{~mm}$ de longueur et d'épaisseur variable suivant le hubiot utilisé ( $1.12 \mathrm{~mm}, 540 \mu \mathrm{m}$ ou $30($ ) [1u1). La paroi plane inférieure est chauffée sur une longueur de $60 \mathrm{~mm}$ par six cartouches résistives insérées dans un bloc en cuivre isolé themsiquement sur sa face arrière. Elles assurent un flux de chaleur maximal en paroi du mini-canal $\varphi$ de l'ordre de $20 \mathrm{~W} / \mathrm{cm}^{2}$. Les vitesses d'écoulement varient de 0.6 à $24 \mathrm{~m} / \mathrm{s}$ et les nombres de Reynolds $R e$, basés sur le diamc̀tre hydraulique $D_{H^{\prime}}$ de 600 à 72000 (tab. I). La nugosité de paroi maximum mesurée est de $2,60 \mu \mathrm{m}\left(R_{t 2}=0,23 \mu \mathrm{m}\right.$ et $\left.R_{z}=2,17 \mu \mathrm{m}\right)$. Les écoulements turbulents considérés ici correspondent au régime lisse des trivaux de Nikuradse.

Tubleau 1. Gummes de vitesse débirante V et de nombre de Reynolds Re pour les trois épaisseurs étudiées.

\begin{tabular}{|c|c|c|c|}
\hline $\begin{array}{l}\text { lipais- } \\
\text { seur du } \\
\text { canal }\end{array}$ & $\begin{array}{l}\text { Diamètre } \\
\text { bydrau- } \\
\text { lique }\end{array}$ & $\begin{array}{l}\text { Gamme } \\
\text { de vilcsise }\end{array}$ & $\begin{array}{l}\text { Gamme de nombre } \\
\text { de Reynolds }\end{array}$ \\
\hline $1.12 \mathrm{~mm}$ & $2,22 \mathrm{~mm}$ & $0,9 \leq V(\mathrm{~m} / \mathrm{s}) \leq 24$ & $2.500 \leq R_{t^{\prime}} \leq 72000$ \\
\hline $540 \mu \mathrm{m}$ & $1.08 \mathrm{~mm}$ & $0.8 \leq V(\pi / s) \leq 18$ & $1400 \leq R^{\prime} \leq 250 \times 0$ \\
\hline 360$) \xi \mathrm{m}$ & $590 \mu \mathrm{m}$ & $0.7 \leq V(\mathrm{~m} / \mathrm{s}) \leq 13$ & $600 \leq R^{\prime} \leq 10(X X)$ \\
\hline
\end{tabular}

IL2 Instrumentation de la boucle et de la cellule d'essai

Un capteur de pression différentielle ${ }^{10}$ mesure les pestes de charge $\Delta p$ aux bornes de la cellule d'essai. Deux débitmètres magnétiques"l sont placés en série. en aval de la pompe. Le premier est dédié aux mesures des petits débits $(0.045 \leq Q(V / s) \leq 0.45)$. le second est utilisé pour les débits plus élevés $(0.45 \leq Q(1 / s) \leq 2)$. Deux sondes de platine $\left( \pm 0,05^{\circ} \mathrm{C}\right)$ mesurent la température de l'eau en amont et en aval de la cellule d'essais (respectivement $T_{\text {tamant }}$ et $T_{\text {arval }}$ ).

Un capteur HFM-7 E/L. développé par VATELL Corp., mesure la température de paroi $T_{p}( \pm 0,5 \%)$ et le flux de chaleur $\varphi( \pm 0,1 \%)$. Il est inséré au centre de la paroi inférieure à $90 \mathrm{~mm}$ de l'entrée du mini-canal et à $25 \mathrm{mrn}$ du début de la zone chauffée. En tenant compte des ordres de grandeur des longueurs d'établissement des couches limites [9. 10, 11 12], on estime que le régime cinématique est établi au niveau du capteur HFM mais que ce n'est pas le cas du régime thernnique dans le cas des canaux de $1,12 \mathrm{~mm}$ et $540 \mu \mathrm{m}$ d'épaisseur.

\section{II.3 Protocole expérimental et traitement des données}

Toutes les mesures ont été faites en régime stationnaire. les paramètres du dispositif expérimental ${ }^{12}$ sont constants pour toute la durée de la mesure d'un point de fonctionnement (une heure). Une fois l'équilibre thermique atteint (40 minutes en moyenne). on acquiert la température de l'eau en amont $T_{\text {umone }}$ et en aval $T_{\text {uval }}$ de la cellule d'essai. le débit $Q$. les pertes de charges $\Delta p$. la température de paroi $T_{p}$

10. $\pm 0.075 \%$ sur la girmme $0.2 \leq \Delta \rho$ (bar) $\leq 20,7$

$11 . \pm 0,5 \%$ de la mesure.

12. Le débil, la puissance électrique injeclée dans les carrouches résistives (o seo w) et la haukur du canal. 
et le flux de chaleur en paroi $\$$. Les séries de mesure sont parcourues dans le sens des débits croissants et décroissants pour détecter un éventuel phénomène d'hystérésis mais aussi aléatoirement pour juger de la reproductibilité des mesures. Le nombre de Nusscit Nu est calculé par :

$$
N_{u}=\frac{\varphi D_{H}}{\lambda\left(T_{p}-\frac{T_{\text {amont }}+T_{\text {aval }}}{2}\right)}
$$

avec $\lambda$ : conductivité thermiqque de l'eau. Le coeflicient de Irottement $C_{f}$ est calculé à partir de $\Delta p$ et d'un bilan des forcess sur une tranche de lluide de longueur $L$ et d'épaisseur $e$ entre deux plaques parallèles :

$$
C_{f}=\frac{2 \tau}{\rho V^{2}}=\frac{\Delta p e}{\rho L V^{2}}
$$

avec $\tau$ : contrainte pariétale, $p:$ masse volumique de l'eau. $V:$ vitesse débitante. Comme base à l'interprétation des résultats expérimentaux, nous utilisons les relations classiques suivantes. Elles concernent (sauf mention contraire) un écoulement entre deux plaques planes dont la distance est suffisamment petite pour que l'on puisse les considérer comme quasi-infinies.

\section{I.3.1 Cas laminaire $\{\operatorname{Re}<2000\}$}

Pour un écoulement établi. le coefficient de frottement $C_{f}$ en paroi s'écrit [13]:

$$
C_{f}=\frac{\tau}{\frac{1}{2} \rho V^{2}}=\frac{24}{R e}
$$

Dans l'hypothèse d'un écoulement cinématiquement et thermiquement établi, on montre facilement que le nombre de Nusselt est constant et vaut 8.24 lorsque le flux de chaleur est uniforme et identique pour les deux parois. Dans le cas où l'une des parois est adiabatique, on obtient $\mathrm{Nu}=5.385$.

\subsubsection{Cas turbulem (régime lisse. $4000<\mathrm{Re}<10^{5}$ )}

On a ici recours ì des corrélations empiriques valables en régime lisse. Pour une conduite de section circulaire classique. le coefficient de perte de charge par frottement $f$, peut être calculé par la relation suivante [13] :

$$
f=\frac{0,3164}{R e^{0.25}}
$$

Le coeffícient de frottement s’écrit alors :

$$
C_{f}=\frac{f}{4}=\frac{0,079}{R e^{0.25}}
$$

Dans le cas d'un écoulement turbulent établi entre deux plans, ie nombre de Nusselt est estimé par la corrélation de Colburn [10] :

$$
N u=0,023 \operatorname{Re}^{0,8} \operatorname{Pr}^{1 / 3}
$$

\section{U.4 Modèle numérique}

En parallc̀le à l'expérimentation. nous avons mené une étude numérique de la veine d'essais à l'aide du code Fluent dans le but d'obtenir des informations qui ne peuvent être obtenues expérimentalement du fait. en particulier. d'une instıumentation limitée à un seul capteur de flux et de température de paroi. L'approche numérique a été préalablement validée à l'aide de cas académiques pour lesquels on dispose de solutions analytiques. Le modèle est une conduite bidimensionnelle dont les paramètres géométriques ${ }^{13}$ et les conditions aux limites ${ }^{\text {it }}$ correspondent à ceux de la veine d'essais. Les calculs sont effectués en régimes stationnaire, laminaire et turbulent. Le traitement des données numériques utilise les rclations données au paragraphe précédent. Seuls les canaux de $1,12 \mathrm{~mm}$ et 300 !:un d'épaisseur ont été modélisés. Dans le cas turbulent, les modèles a $k-\varepsilon$ stundard $» \mathrm{ct}$ «bas Reynolds» on tété utilisés.

\section{RÉSULTATS ET DISCUSSION}

\section{III.I Coefficient de frottement}

La figure / présente l'évolution du coefficient de frottement $C_{\mathrm{r}}$ en fonction du nombre de Reynolds Re. Les résultats expérimentaux sont globalement similaires à ceux obtenus en 2000 [1]. En régime laminaire. les mesures correspondant les canaux de $540 \mu \mathrm{m}$ et $300 \mu \mathrm{m}$ d’épaisseur suivent la tendance en $/ / R e$ de l'équation 3 mais elles se positionnent à des valeurs supérieures. l'écart augmentant lorsque le diamètre hydraulique diminue. Ces observations sont en accord avec les conclusions des études présentées en introduction $[2,4,7]$

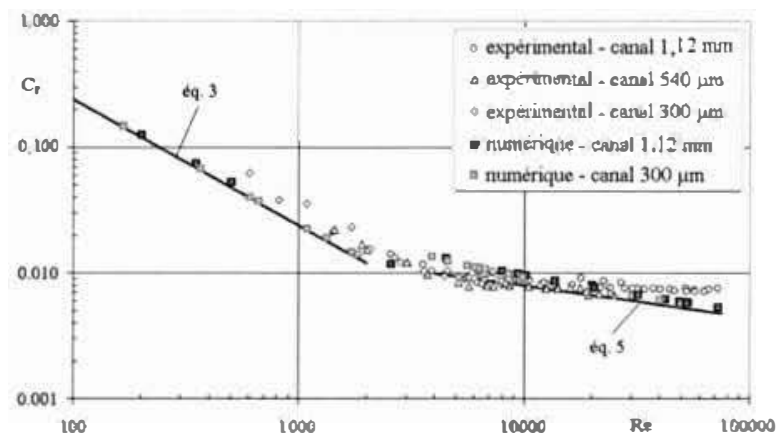

Figure 1 : Distribution du coefficient de frottement $C_{f}$ en fonction du nombre de Reynolds Re (résultats expérimentaux et numériques).

En régime turbulent, les mesures sont du même ordre de grandeur que celui donné par l'équation 5. Cependant elles ne suivent pas l'évolution en $R^{-0.25}$, typique du régime lisse. mais s'orientent plutôt vers une loi de comportement peu dépendante du nombre de Reynoids $\left(R e^{-0.045}\right)$, compostement

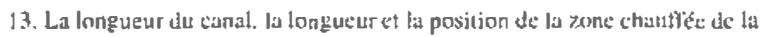
parni inféricurc. Lu haurcur du canal. la position du capteur fIFM.

14. La vilesse débitante $\nvdash$. tc flux de chaleur el la tcmpérature de l'eau 
caractéristique du régime rugueux. Notons que Acosta ef al. [7] ont également observé cette tendance dans leurs canaux dont les rugosités semblent correspondre ì notre veine d'essai. Les résultats numériques. très proches des corrélations classiques en régimes laminaire et turbulent. ne confirment pas ces mesures. Ce phénomène peut s'expliquer en meltant en parallèle les résultats obtenus par Acosta el al. [7] dans le canal ayant un état de surface soigné («poli optique ) et nos tésultats numériques. Ces deux cas présentent en effet des états de surface similaires dans la mesure où. par défaut. Fluent ne modélise pas les rugosités de parvi et utilise un coefficient calibré pour faire correspondre le modèle $k$ - $\varepsilon$ au régime turbulent lisse. On peut en déduire que les rugosités mesurées dans notre veine d'essai, bien que correspondant au régime lisse de Nikuradse. peuvent être responsables des déviations en régime turbulent. Le modèle numérique sera prochainement complété par la prise en compte des rugosités.

\section{IlJ.2 Nombre de Nusselt}

Les mesures. représentées sur la figure 2, font apparaître des évolutions de $N ı$ en fonction de $R e$ similaires pour les trois épaisseurs étudiées sur leur gamme de $R e$ respectives. On distingue en effet trois plages de $R e$ sur lesquelles $N u$ a des componements différents. la taille et la position de celles-ci sur la gamme de $R e$ étant différentes pour chaque épaisseur (tab. 2).

Tableau 2. Plages des nombres de Reynolds correspondants aux différents comportement du nombre de Nusselt pour les irois épaisseurs éludices.

\begin{tabular}{|c|c|c|c|}
\hline & Canal $1.12 \mathrm{~mm}$ & Canul $5.411 \mathrm{~mm}$ & (ana1 301) $\mu \mathrm{m}$ \\
\hline $\begin{array}{c}\text { Zone } \\
1\end{array}$ & $26(X)<-R e<7000$ & $1+(x)<\operatorname{Re}<5(x)$ & $\left.\mathrm{G}(\mathrm{x})<R_{e}<3 \mathrm{OK}\right)$ \\
\hline $\begin{array}{c}\text { Zanse } \\
2\end{array}$ & $7(x)()<R e<3\})(x) 0$ & $5000<R_{e}<17000$ & $3000<\operatorname{Re}<6(000)$ \\
\hline $\begin{array}{c}\text { Zone } \\
3\end{array}$ & $200\left(X_{0}\right)<R_{e}<72000$ & $17(x)(j)<\overline{R_{t}}<25(x)$ & $6(0)<R_{t^{\prime}}<10(x) 0$ \\
\hline
\end{tabular}

La première zone correspond au regıme laminaire. On observe le même compostement de $\mathrm{Nu}$ que celui rapporté par Wang \& Peng [5]. à savoir l'augmentation de $\mathrm{Nu}$ avec $R e$ alors que la théorie prévoit une valeur constante. Dans notre cas. l'étude numérique de la veine d'essais a montré que ce phénomène est dû au non-établissement du régime thermique au niveau du capteur HFM en écoulement laminaire. Pour les trois épaisseurs. le nombre de Nusselt suit la même tendance en $R e^{0.45}$ mais ses valeurs tendent à augmenter avec l'épaisseur du canal. Les résultats numériques suivent également celte évolution de $\mathrm{N} u$ avec $R e$ mais avec des valeurs supérieures aux mesures.

La zone 2. située aux nombres de Reynolds intennédiajres. correspond à la transition laminaire-lurbulent. Les signaux observés sur l'oscilloscope font en effet apparaître des augmentations quasi-instantanées du flux de chaleur. la température de paroi diminuant simultanément. Ces fluctuations correspondent probablement au passage de bouffées turbulentes qui augmentent soudainement le coefficient d'échange thermique. La fréquence de ces bouffées augmente avec le nombre de Reynolds tandis que l'amplitude des fluctuations de flux diminue progressivement jusqu'à ce que la turbulence soit pleinement développée au niveau du capteur HFM el que les moyennes temporelles des deux signaux se stabilisent de nouveau. Pour les trois canaux. cette zone apparầt et se prolonge bien au-delà des valeurs classiques de $\operatorname{Re}(2300-4000)$. Le tableau 3 résume les gammes de nombre de Reynolds. basés sur le diamètre hydraulique. dans lesquelles ont été observées les fluctuations de transfen thermique pour chacune des trois épaisseurs. Nous y avons également porié le nombre de Reynotds basé sur l'abscisse $x=90 \mathrm{~mm}$ correspondant à la distance entre le capteur HFM é l'entrée du canal. Les valeurs cor. respondantes sont caractéristiques des nombres de Reynolds critiques observés en couche limite ${ }^{15}$. A titre de référence, nous avons indiqué les épaisseurs typiques de couche limite $\delta_{01,99}$ à $99 \%$ en régime laminaire ( $\mathrm{Eq} .7[9]$ ). S`il est clair que les couches limites se développant sur les deux parois

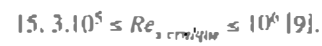

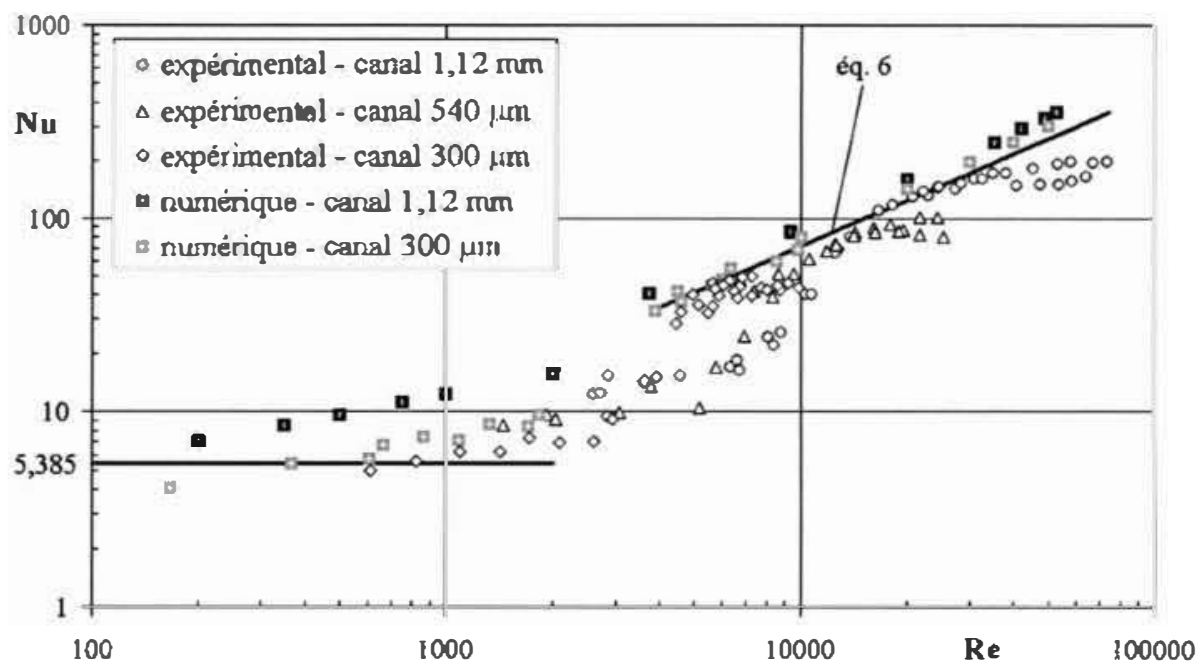

Figure 2 : Distribution du nombre de Nusselt Nu en fonction du nombre de Reynolds Re (résultats expérimentaux et numériques). 
Tubleas 3. Nombres de Reinolds de iransision pour les trois épaisseurs éludiées.

$\begin{array}{cccc}\begin{array}{c}\text { Epais- } \\ \text { seur du } \\ \text { canal }\end{array} & \begin{array}{c}\text { Re de transi- } \\ \text { tion busć sur } \\ \text { le diamètre } \\ \text { hydraulique }\end{array} & \begin{array}{c}\text { Re de transition } \\ \text { hasc sur } \\ x=90 \mathrm{~mm}\end{array} & \begin{array}{c}\text { Epaisseur de } \\ \text { couche limite } \\ \text { laminaire cor- } \\ \text { respondante }\end{array} \\ 300 \mu \mathrm{m} & 3000-4500 & 4.0^{5}-6.8 .10^{5} & 550-670 \mu \mathrm{m} \\ 540 \mu \mathrm{m} & 7000-13000 & 5.8 .10^{5}-1.1 .10^{6} & 430-590 \mu \mathrm{m} \\ 1120 \mu \mathrm{m} & 7000-16000 & 2.8 .10^{5}-6.4 .10^{5} & 560-850 \mu \mathrm{m}\end{array}$

opposées se sont effectivement réunies au point de mesıre pour le canal le plus mince. c’est à peine le cas pour le canal de $1.12 \mathrm{~mm}$. Dans ce dernier cas, nous pouvons dire, en premic̀re approximation, que l'écoulemenı laminaire esı plus proche d'un écoulement de couche limite que d'un écoulemenı élabli en conduite.

$$
\delta_{0,9 y} \cong \frac{5 x}{\sqrt{R e_{x}}}
$$

Il convient de noter que ces gammes de Reynolds de transition ne s'accordent pas avec les observations faites sur les ligures relatives au coeflicient de frottement. Ceci est probablement lié au fait que le nombre de Poiseuille résulte d'une mesure globale de perte de charge alors que le nombre de Nusselt traduit une mesure locale de transfen thermique. La visualisation des couches limites par injection de fluorescéine est en ce moment à l’élude. Il faut noter entin que l'étude numérique menée ici ne permet pas de prévoir le changement de régime de l'écoulement.

La zone 3. correspondant à la turbulence développée, se situe aux grands nombres de Reynolds. Pour les trois épaisseurs éludiées. le nombre de Nusselt est proche des valeurs issues de l'équation 6 de sorte que les mesures ne mettent pas en évidence un effet du diamètre hydraulique sur le coefficient convectif. Nous remarquons également sur la figure 2 que le nombre de Nusselt suil la lendance en $R^{0.8}$ de la relation 6 avant de dévier progressivement vers des valeurs inférieures ${ }^{\prime A}$. Celle dernière remarque peut s'expliquer par les effets conjugués de (1) la dissipation visqueuse volumique sur la mesure de la température de paroi comple tenu des vitesses d'écoulement élevées el (2) d'un défaut d'uniformité du flux de chaleur à la surface du mini-canal. La dissipation visqueuse volumique esı responsable de l'élévation de la température de paroi entraînant l'augmentation de $\Delta T=T_{p-}\left(T_{\text {ammont }}+T_{\text {aiaf }}\right) / 2$ ct ainsi la diminution de $N_{u}$. Nous avons vérilié expérimentalement que la prise en comple de la dissipation visqueuse lors du traitement des mesures réduit l'écart entre le nombre de Nusselt et les valeurs données par la relation 6 de moitié, ce point étant confirmé par l'étude numérique. Celle dernière a également mis en évidence l'effet de la variation spatiale du flux de chaleur qui conduirait à sous-estimer le flux moyen à la paroi et, par conséquent, le nombre de Nusselt. Notons que ce phénomène. imputable à notre système de chauffage, esı probablement accentué par le mécanisme de montage du capteur HFM sur le bloc chauffant.

16. Jusqu'à-45\% prut Re $=7.110^{4}$

\section{D CONCI,USIONS}

Les résultats de celle élude ne sonı pas de nalure à remettre en cause la validité des lois classiques dans le cas des mini-canaux bidimensionnels d'épaisseur au moins égale à $0.3 \mathrm{~mm}$. Nous avons montré que si des déviations sont observées par rappon aux corrélations classiques. elles sont généralement à attribuer à des effets classiques du type régimes cinématique et thermique non établis ou à des défauts du dispositif expérimental tels qu un défaut d'uniformité du flux thermique. La déviation du coefficient de frottement en régime turbulent peut avoir pour origine l'état de surface de nos canaux. Les résultats de celle élude ne mettent pas en évidence un effel du diamètre hydraulique sur les Iransferts thermiques différent de celui prévu par les corrélations classiques du type "Colhurn".

\section{BIBLIOGRAPHIE}

11] Debray [i.. Franc J.-P.. Maitre T.. Rerxald S. (2001). "Mesure des coefficients de transferts thermiques par conveclion forcée en mini-canaux ». Mécanique et Industrie. 2. 443. 454.

|2| Rahмa: M.M. (2000). - - Measurements of heal Iransfer in microchannels heat sinks ». Int. Comm. Heat Mass Transfer. Vol. 27. No. 4. 465-506.

|3| Adans T.M., Agejl-Khaljk S.I.. Jeter S.M.. Qureshi Z.H. (1997). - * An experimental investigation of single phase forced convection in microchannels $n$. Int. J. Heat Mass Transfer. Vol. 41. No. 6-7. 851-857.

[4] Gao P., Leferson S., Favre-Marinet M. (2002). - « Scale effects on hydrodynamics and heat Iransfer in Iwo-dimensional mini and micruchannels n. Int. J. of Thennal Sciences. Vol. 41, $1017-1047$

[5] WANG B.X.. PI:NG X.F. (1994). - e Experimental investigation on licquid forced-convection hcal transfer through nicrochannels ». Imr. J. Hear Mass Trumsfer, Vol. 37 , Suppl. 1,73-82.

|6| PENG X.F.. PEvlikson G.P. (1996) — "Conveclive heat Iransfer and flow friction for water flow in nicrochannels structures ». Int. J. Heat Mass Transfer. Vol. 39, No, 12, 25992608 .

[7] Acosta R.E.. MULU:R R.H.. TOBIAS C.W. (1985). * Transport processes in narrow (capillary) channels ". A/ChE Journal, Vol. 31. No. 3. $\$ 73-482$

[81 Zejghami R., Lnser D.. Zhou P.. Asheghi M.. Devanisetypathy S., KenNi T., Santingo J.. Gundoson K. (2000). * Experimental invextigation of flow transition in microchan nels using micron-resolution particle image velocimety $»$. Int Soc. Conf. on Themsal Phe'nomena, j48-153.

[9] Schlicitixg H. (1960). - * Boundaty layer theory ». 4th Edition McGraw-Hill.

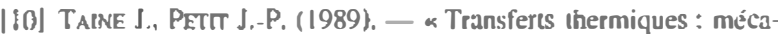
nique des tluides anjsolhermes ». Dunod Université

[II] Com(t.IT R. (1963). - " Mécanique expérintentale des fluides s. Tome 2, Masson \& Cic.

(12] Chassaing P. (2000). - « Mécanique des fluides ». $2^{e}$ édition. Collection Polytuch.

|13| IDEL'CIK 1.E. (1986) ^ Mémenıo des pertes de charge s. Eyrolles. Collection de la Direction des Eludes el Recherches d’Electricilé de Jirance. 